\title{
A genome-wide investigation of microsatellites mismatches and the association with body mass among bird species
}

\author{
Haiying Fan ${ }^{\text {Corresp., }}{ }^{1}$, Weibin Guo ${ }^{1}$ \\ ${ }^{1}$ Department of Ecology, College of Life Sciences, Wuhan University, Wuhan, China \\ Corresponding Author: Haiying Fan \\ Email address: fanhaiying1989@126.com
}

Mutation rate is usually found to covary with many life history traits of animals such as body mass, which has been readily explained by higher number of mutation opportunities per unit time. Although the precise reason for the pattern is not yet clear, to determine the universality of this pattern, we test whether life history traits impact another form of genetic mutation, the motif mismatches in microsatellites. Employing published genome sequences from 65 avian species, we explore the motif mismatches patterns of microsatellites in birds on a genomic level and assess the relationship between motif mismatches and body mass in a phylogenetic context. We have found that small-bodied species have a higher average mismatches and we suggest that higher heterozygosity in imperfect microsatellites lead to the increase of motif mismatches. Our results obtained from this study, suggest that a negative body mass trend in mutation rate may be a general pattern of avian molecular evolution. 
1 A genome-wide investigation of microsatellites mismatches and the association with body

2 mass among bird species

\section{Haiying Fan $^{1}$ \& WeibinGuo ${ }^{1}$}

$4 \quad{ }^{1}$ Department of Ecology, College of Life Sciences, Wuhan University, Wuhan 430072, China

5 Corresponding author: Haiying Fan, email: fanhaiying1989@126.com

6 Department of Ecology, College of Life Sciences, Wuhan University, Wuhan 430072, China

7

9

10

11

12

13

14

15

16

17

18

19

20

21

22

23

24

25

26

27

28

29

30 


\section{Abstract}

34 Mutation rate is usually found to covary with many life history traits of animals such as body

35 mass, which has been readily explained by higher number of mutation opportunities per unit time.

36 Although the precise reason for the pattern is not yet clear, to determine the universality of this

37 pattern, we test whether life history traits impact another form of genetic mutation, the motif

38 mismatches in microsatellites. Employing published genome sequences from 65 avian species,

39 we explore the motif mismatches patterns of microsatellites in birds on a genomic level and

40 assess the relationship between motif mismatches and body mass in a phylogenetic context. We

41 have found that small-bodied species have a higher average mismatches and we suggest that

42 higher heterozygosity in imperfect microsatellites lead to the increase of motif mismatches. Our

43 results obtained from this study, suggest that a negative body mass trend in mutation rate may be

44 a general pattern of avian molecular evolution. 


\section{Introduction}

46 It has long been recognized that the molecular evolutionary rates always covary with many life

47 history traits of animals. Numerous studies have documented a negative relationship between the

48 rate of molecular evolution and body mass (Nabholz, Glémin \& Galtier, 2008; Bromham, 2011;

49 Amos \& Filipe, 2014), where genes in small-bodied species are likely to evolve faster than those

50 in large-bodied species. This has been readily explained by higher number of mutation

51 opportunities per unit time (generation length hypothesis, Li et al., 1996) or higher mutation

52 probability in a round of DNA replication due to higher metabolic rate (metabolic rate hypothesis,

53 Mindell et al., 1996) in small-bodied species. Although the precise reason for the pattern is not

54 clear at present, to determine the universality of this pattern, we need to study additional form of

55 genetic mutation besides mitochondrial DNA or nuclear 'genes' which are most frequently used.

56 The first to consider is the fastest evolving components of the genome such as microsatellites.

57

58 Microsatellites, also known as simple sequence repeats (SSRs), are tandem repeats of simple

59 nucleotide motifs, which have wide coverage in eukaryotic and prokaryotic genomes (Tóth,

60 Gáspári \& Jurka, 2000; Ellegren 2004; Adams et al., 2016). One feature of microsatellites is that

61 they have a high mutation rate $\left(10^{-7}\right.$ to $10^{-3}$ mutations per locus per generation), leading to high

62 heterozygosity and extensive length polymorphisms (Kruglyak et al., 2000). It has long been

63 assumed that the major cause of variation of microsatellite repeats is replication slippage

64 (Kornberg et al., 1964; Bhargava \& Fuentes, 2010), which will increase or decrease repeat copy

65 numbers in microsatellites. Specifically, when it creates a loop in one of strands, a slippage error

66 occurs. If the loop is formed in the replicating strand, it will introduce an insertion. If the loop is

67 in the template strand, a deletion will emerge. Several mathematical models of microsatellite

68 evolution have been proposed to represent the mutation processes of microsatellites, such as

69 stepwise mutation model (SMM) of Ohta \& Kimura (1973), which suggests that mutation in

70 microsatellite loci occurs by one repeat unit at a time.

71

72 Many studies on microsatellites have explored the frequencies, abundance and polymorphism of

73 microsatellites in the genomes (Wang et al., 2014; Qi et al., 2015; Adams et al., 2016). Few, if

74 any, have correlated these microsatellite characters to the life history traits of a species.

75 Specifically, microsatellites are hypothesized to experience a life cycle: start short (birth) and 
76 expand predictably due to mutation bias (expansion) until they become unstable and either 77 collapse or degrade through internal point mutations (contraction and death) (Chambers \& 78 MacAvoy, 2000; Buschiazzo \& Gemmell, 2006). Life history traits of species are expected to 79 have an influence on the life cycle - 'birth', expansion, and 'death' —of microsatellites in the 80 genome (Amos \& Filipe, 2014). For example, in smaller species, higher mutation rate allows the 81 'birth' and expansion of microsatellites faster, due to higher mutation rate and slippage rate.

82 Since the death rate is lower than the birth rate, microsatellites tend to accumulate in the genome 83 (Buschiazzo \& Gemmell, 2006). In that, the smaller species harbour a higher frequency of 84 microsatellites across the genome, which has been proved in mammals (Amos \& Filipe, 2014). 85

86 It is well known that except for repeat copy number variation, a microsatellite (e.g.

87 ATATATATAT) also suffers from nucleotide substitutions and insertion/deletion mutations, 88 hence becoming imperfect (e.g. ATATATCATAT: AT repeat with an insertion of C). Perfect 89 and imperfect microsatellites are thus defined. It has been found that genomes possess a 90 relatively small but significant number of imperfect microsatellites (Brinkmann et al., 1998).

91 Mismatch variation of imperfect microsatellites is critical for their maintenance in the genome 92 and imperfect microsatellites are more stable compared to perfect microsatellites since the 93 former is less prone to slippage mutation (Sturzeneker et al., 1998). Several previous studies

94 have already revealed the genome-wide motif imperfection pattern among species (e.g. Behura \& 95 Severson, 2015). Nevertheless, our understanding of motif mismatches in imperfect

96 microsatellites is still very limited and their correlation with life history traits remains to be 97 revealed and explained.

99 In this study, we used 65 avian genome sequences, employing SciRoKo (Kofler, Schlötterer \& 100 Lelley, 2007) to search SSRs in the whole genome. We chose avian genomes for this study 101 because microsatellites have been widely used in population genetics of bird species, yet the 102 pattern of microsatellites mismatches in birds is still not well understood, mostly owing to the 103 lack of avian genomic information. With the advance of whole genome sequencing, evolution of 104 microsatellites is attracting attention from researchers. With the genome-wide microsatellites 105 data in hand, we presented the first detailed comparative study of microsatellites, aiming to 
106 reveal the patterns of motif mismatches across different bird species and to help understand the

107 relationship with life history traits.

108

109 Materials \& Methods

110 Genome sequences and body mass

111 We downloaded fasta files of the 65 avian genomes from NCBI

112 and GigaDB (http://dx.doi.org/10.5524/101000). These avian species represent nearly all of the

113 major clades of living birds. We compiled data from the original and secondary references and

114 the world-wide web about the mean body mass of adult males and females (Table S1). If a mean

115 value was not provided for a species, we took the median of the range. Where separate body

116 masses were given for males and females, the average value of the masses was calculated.

117

118 Identification of microsatellites

119 We searched microsatellites in each genome sequences using SciRoKo 3.4, a simple sequence

120 repeats (SSRs) identification program (Kofler, Schlötterer \& Lelley, 2007), with the default

121 parameters (minimum score $=15$ and mismatch penalty $=5$ ) in the mismatched modes. In

122 addition, we used different parameters to search SSRs (minimum score $=15$ and mismatch

123 penalty $=3$, minimum score $=10$ and mismatch penalty $=5$ ) considering changing in parameters

124 would affect the results of this study. Specially, the motif mismatches refer to the number of base

125 mismatches of an imperfect microsatellite compared with its idealized perfect counterpart. For

126 example, the string TACTACTAGTACTAC, is a trinucleotide repeat with five repeats and, by

127 comparison with its idealized perfect counterpart (consensus repeat), it has a mismatch of 1 . The

128 number of mismatches of each microsatellites as well as their length for each genome was used

129 for different comparative analyses across the species.

130

131 Statistical analysis

132 In this study, we used phylogenetic generalized least-squares regression (PGLS) (Freckleton,

133 Harvey \& Pagel, 2002) implemented in the package 'ape' (Paradis, Claude \& Strimmer, 2004) to

134 control shared ancestry (for the script used, see Figure S1). We used the evolutionary tree of the

13548 bird species estimated by Jarvis et al. (2014) as a backbone topology, and used the

136 phylogenetic information provided by Jetz et al. (2012) to add the remaining 17 species (for the 
137 resulting phylogeny, see Figure S2). In order to achieve the statistical requirements for linearity

138 and normality, adult average mass were $\log 10$-transformed prior to analysis. Average

139 mismatches was reciprocal transformed. GC content was arcsine square root transformed.

140

141 Firstly, we computed some basic statistics on characteristics of microsatellite loci in 65 bird

142 genomes (Table S2, S3, S4, S5). Secondly, to better understand the occurring of motif

143 mismatches in bird genomes, we determined the frequency of microsatellites of $20 \mathrm{bp}$ that either

144 lacks mismatch or harbours one mismatch for each species (Table S6). 20bp was used because

145 that the average length of perfect microsatellites of 65 birds is $20 \mathrm{bp}$. Then we computed the ratio

146 of imperfect (mismatch $=1)$ repeats frequency to perfect $($ mismatch $=0)$ ones. Then, we

147 employed a PGLS, treating the ratio of imperfect (mismatch $=1)$ to perfect (mismatch $=0$ )

148 repeats as a dependent variable and body mass as an explanatory variable. Thirdly, we explored

149 whether or not the extent of motif mismatches is related to genomic abundance of imperfect

150 microsatellites. We first calculated the probability of per-site mismatch (the total number of

151 mismatches divided by total lengths of all loci) in each genomes. Then the expected number of

152 mismatches was determined based on the length and compared with the observed number of

153 mismatches in each imperfect microsatellite (Table S7). The first paired sample $t$-test was

154 conducted between the numbers of microsatellites harbouring more mismatches than expected

155 and that of carrying fewer mismatches than expected. The second paired sample $t$-test was

156 performed between imperfect repeats that have a length of at least 30 bp and have either $<3$ or $\geq 3$

157 mismatches (Table S8). Finally, to test whether differences of mismatches in imperfect

158 microsatellites link with body mass, we fitted a PGLS analysis with average mismatches of

159 imperfect SSRs as dependent variable and body mass as a predictor. The average mismatches of

160 imperfect SSRs in individual genomes was estimated as the sum of mismatches divided by the

161 number of imperfect microsatellites (Table S1). Average mismatches was used because it

162 indicates the mismatches in an 'average' imperfect SSR. For controlling the probability that GC

163 content will have a potential influence on microsatellite mismatches, we added it to the models

164 as a predictor variable. Taking di-, tri-, tetra-, penta- and hexamers as the five classes of repeats,

165 we repeated the PGLS analysis in each repeat type. Since the mutations in the mononucleotide

166 repeats tend to cause the emergence of a new motif of other repeat type, we excluded it from our

167 analysis. All statistical analyses were conducted with R 3.1.2 (R Development Core Team 2014). 


\section{Results}

170

171 (a) Characteristic of microsatellite loci in 65 avian species

172 In total, 11803896 SSR loci with a minimum length of 15 bp were identified from 65 avian

173 genome assemblies, and were classified into mono-, di-, tri-, tetra-, penta- and hexanucleotide

174 SSRs according to the motif length (Table S2). Among these, mononucleotide SSRs are the most 175 abundant (42.3\%) type, followed distantly by tetra- (18.8 \%) and pentanucleotide SSRs (17.1\%)

176 (Table S2; Figure S3). The SSR abundance composition and SSR density of the birds varies

177 greatly among species, with the maximum value in Anas platyrhynchos (416040 counts; 376.49

178 counts/Mb) and the minimum value in Melopsittacus undulatus (81643 counts; 73.07 counts/Mb).

179 Additionally, the SSR abundance composition are predicted by genome size $(\beta \pm S E=1.56 \pm$

$\left.1800.64, t=2.44, P=0.017, R^{2}=0.09\right)$.

181

182 (b) Frequency of imperfect microsatellites in bird genomes

183 The number of imperfect microsatellites varies among the birds species and the imperfect repeats

184 account for $15-27 \%$ of all microsatellites searched from the genome assemblies of the 65 bird 185 species as shown in Table S3. The imperfect repeats represented less than $0.2 \%$ of the genome 186 sequence in most of these birds except four species (Anas platyrhynchos, Calypte anna, Columba

187 livia, Picoides pubescens) (Figure S4). The data in Table S3 and Figure S4 shows that the 188 frequency of imperfect microsatellites in bird genomes appears substantial variation among these 189 species. It was observed that Anas platyrhynchos has a higher percentage of imperfect 190 microsatellites than other bird species. Moreover, the proportion of imperfect repeats varies 191 differentially among species, to some extent, depending on the motif size of microsatellites. 192 Specifically, the paired sample $t$-test results indicated the di-, tri- and hexanucleotide SSRs have 193 an increased rate of motif mismatches compared with all other types of motif size (Table S4).

194 Furthermore, it seems that this pattern is conserved among different avian species.

195

196 (c) The occurring of motif mismatches

197 Imperfect microsatellites are longer than perfect microsatellites in each species ( $37 \mathrm{vs} 20 \mathrm{bp} ; t=$ 198 33.334, $d f=64, p<0.001$; Table S5). The PGLS analysis revealed that the small-bodied species 
199 has a higher ratio of imperfect $($ mismatch $=1)$ to perfect $($ mismatch $=0)$ repeats of $20 \mathrm{bp}$ than

200 large-bodied species $\left(\beta \pm S E=-0.006 \pm 0.002, t=2.86, P=0.006, R^{2}=0.12\right)$.

201

202 (d) The accumulation of mismatches in imperfect microsatellites and genomic abundance

203 The paired sample $t$-test revealed that the microsatellites harboring mismatches higher than 204 expected has significantly lower abundance than that carrying mismatches lower than expected

205 (13381 versus 25138 counts; $t=22.651, d f=64, p<0.001$; Table S7), implying that the

206 imperfect microsatellites who containing more mismatches have lower abundance in the genome.

207 We also found that loci with three or more number of mismatches are less common than that

208 have less than three mismatches (7364 vs 18361 counts; $t=11.316, d f=64, p<0.001$; Table S8).

209

210 (e) correlation between body mass and average motif mismatches

211 We found that on a whole genome scale, the average body mass accounts for $28.2 \%$ of the

212 variation in average mismatches of imperfect SSRs (Table 1, Figure 1). Body mass also has a

213 significantly negative correlation with microsatellites mismatches in five motif length classes

214 (Table 1, Figure 2). This negative correlation remains significant when adding GC content to the

215 regression models. Inclusion of GC content only enhances the model's explanatory power

216 slightly except in tetra- and pentanucleotide SSRs. When we used different parameters including

217 minimum score 15 and mismatch penalty 3 and a minimum score of 10 and mismatch penalty 5

218 to search microsatellites in the genomes, the results of repeated analyses were highly consistent

219 (Table S9). This confirmed that our observations were not influenced by the search parameters of 220 microsatellites.

221

\section{Discussion}

223

224 In the present study, we did genome-wide search of microsatellites using SciRoKo with the same 225 parameters to ensure that the program can search all possible microsatellites with the same 226 probability for every genome. Microsatellites search results showed that the frequency of 227 microsatellites varies extensively among species. We have also found a positive relationship 228 between microsatellites abundance and genome size among 65 bird species, which is consistent 229 with earlier studies (e.g. Hancock, 1996). After providing a general description of the basic 
230 characteristics of microsatellites, we particularly focused on comparing the motif mismatches of

231 imperfect microsatellites to body mass across bird species in a phylogenetic context.

232

233 We found a negative relationship between body mass and the ratio of frequency of imperfect

234 repeats $($ mismatch $=1)$ to perfect (mismatch $=0$ ) ones with the same length $20 \mathrm{bp}$ among the

235 species. Moreover, it is known that mutations in microsatellites shorter than a critical length are

236 generally gain or loss of single repeat units which cannot disturb the repeat tract (Buschiazzo \&

237 Gemmell, 2006). Whereas when it reached a critical length, mismatch was introduced, a perfect

238 microsatellite became imperfect. Here, our result implied that the introduction of motif

239 mismatches in imperfect microsatellites is significantly associated with the nature of point

240 mutation in microsatellites. In small-bodied species, since more perfect microsatellites suffer

241 from the introduction of mismatches due to the higher mutation rate, larger number of imperfect

242 microsatellites relative perfect ones can be observed.

244 We observed that the microsatellites harbouring mismatches higher than expected have lower

245 abundance than that carrying mismatches lower than expected. Consistent with this result, we

246 also found that the microsatellites $\geq 30$ bp and $>3$ mismatches have lower abundance than that

$247 \geq 30 \mathrm{bp}$ and $<3$ mismatches, indicating that mismatches of motifs is a key determinant leading to

248 a paucity of long imperfect microsatellites in the genome. That is to say, mismatches would

249 stabilize the repeat array and impede the further expansion. When the extent of mismatches

250 reached saturation point, the repetition pattern is interrupted, leading the microsatellites to

251 degeneration and death. (Taylor, Durkin \& Breden, 1999; Harr \& Schlotterer, 2000; Yamada et

252 al., 2002; Vowles \& Amos, 2006). Although the exact details of death is still poorly understood,

253 the relative number of older mismatches in an 'average' microsatellite is likely to reflect the

254 mutability during its lifetime. It can be further confirmed by the finding that the average

255 mismatches of imperfect SSRs decreases with increasing body mass.

256

257 Our results that higher average mismatches of imperfect SSRs in small-bodied species support a 258 correlation between mutation rate and life history traits. The pattern is usually explained by a

259 generation length model, where smaller species evolve faster due to higher number of mutation opportunities per unit time (Li et al., 1996). In addition, body mass might affect the mutation rate 
261 through a link with metabolic rate and/or body temperature, which can directly change the

262 mutation probability in a round of DNA replication (Mindell et al., 1996). Apart from these two

263 key hypotheses, a rising hypothesis which proposes mutation rates are influenced by

264 heterozygosity (Amos, 2010) can better explain the intrinsic correlation of motif mismatches

265 with body mass. Smaller species have larger number of imperfect microsatellites which has been

266 demonstrated by our data $\left(\beta \pm S E=-0.008 \pm 0.002, t=4.008, P<0.001 ; R^{2}=0.203\right)$. Meanwhile,

267 more heterozygous sites at these imperfect microsatellites can be expected. Recognition and

268 'repair' of heterozygous sites during synapsis will cause additional rounds of DNA replication

269 which in turn provide more opportunities for mutations (Amos, 2011) and introduce more motif

270 mismatches at imperfect microsatellite sites. Therefore, a negative relationship between body

271 mass and motif mismatches can be observed. We suggest that heterozygote instability hypothesis,

272 which is supported by increasing evidence (Drake, 2007; Masters et al., 2011; Amos, 2013;

273 Amos, 2016), could provide a potential link between body mass and motif mismatches. However,

274 further studies are needed in order to examine carefully whether homologous imperfect

275 microsatellites are generally more prone to introduce mismatches in smaller species with a detail

276 comparison between sister species.

277

278 Conclusions

279

280 In conclusion, the present study is the first effort to explore the motif mismatches patterns of

281 microsatellites in birds on a genomic level. Our results obtained from this study provide support

282 for the long-standing correlation between mutation rate and life history traits and suggest that a

283 negative body mass trend in mutation rate may be a general pattern of avian molecular evolution. 284

285 Acknowledgements

286 We thank Xin Lu, Hongtao Xiao, Guoyue Zhang, Changcao Wang, Qingchen Zhang and

287 Juanjuan Rao for data collection, statistical advice and insightful discussions. We also thank

288 William Amos and Andrew Clarke for helpful suggestions and two anonymous referees for 289 comments on earlier versions of this manuscript.

\section{References}


292

293

294

295

296

297

298

299

300

301

302

303

304

305

306

307

308

309

310

311

312

313

314

315

316

317

318

319

320

321

Adams RH, Blackmon H, Reyes-Velasco J, Schield DR, Card DC, Andrew AL, Waynewood N, Castoe TA. 2016. Microsatellite landscape evolutionary dynamics across 450 million years of vertebrate genome evolution. Genome 59:295-310 DOI 10.1139/gen2015-0124.

Amos W. 2010. Heterozygosity and mutation rate: evidence for an interaction and its implications. BioEssays 32:82-90 DOI 10.1002/bies.200900108.

Amos W. 2011. Population-specific links between heterozygosity and the rate of human microsatellite evolution. Journal of Molecular Evolution 72:215-221 DOI 10.1007/s00239010-9423-2.

Amos W. 2013. Variation in heterozygosity predicts variation in human substitution rates between populations, individuals and genomic regions. PLOS ONE 8:e63048 DOI 10.1371/journal.pone.0063048.

Amos W, Filipe LNS. 2014. Microsatellite frequencies vary with body mass and body temperature in mammals, suggesting correlated variation in mutation rate. PeerJ 2:e663 DOI 10.7717/peerj.663.

Amos W. 2016. Heterozygosity increases microsatellite mutation rate. Biology Letters 12: 20150929 DOI org/10.1098/rsbl.2015.0929.

Behura SK, Severson DW. 2015. Motif mismatches in microsatellites: insights from genomewide investigation among 20 insect species. DNA Research 22:29-38 DOI 10.1093/dnares/dsu036.

Bhargava A, Fuentes FF. 2010. Mutational dynamics of microsatellites. Molecular Biotechnology 44:250-266 DOI 10.1007/s12033-009-9230-4.

Brinkmann B, Klintschar M, Neuhuber F, Huhne J, Rolf B. 1998. Mutation rate in human microsatellites: influence of the structure and length of the tandem repeat. American Journal of Human Genetics 62:1408-1415 DOI 10.1086/301869.

Bromham L. 2011. The genome as a life-history character: why rate of molecular evolution varies between mammal species. Philosophical Transactions of The Royal Society BBiological Sciences 366:2503-2513 DOI 10.1098/rstb.2011.0014.

Buschiazzo E, Gemmell NJ. 2006. The rise, fall and renaissance of microsatellites in eukaryotic genomes. BioEssays 28: 1040-1050 DOI 10.1002/bies.20470. 
322

323

324

325

326

327

328

329

330

331

332

333

334

335

336

337

338

339

340

341

342

343

344

345

346

347

348

349

350

351

Chambers GK, MacAvoy ES. 2000. Microsatellites: consensus and controversy. Comparative Biochemistry and Physiology B-Biochemistry \& Molecular Biology 126: 455-476 DOI 10.1016/S0305-0491(00)00233-9.

Drake JW. 2007. Too many mutants with multiple mutations. Critical Reviews in Biochemistry and Molecular Biology 42:247-258 DOI 10.1080/10409230701495631.

Ellegren H. 2004. Microsatellites: simple sequence with complex evolution. Genetics 5: 435445 DOI $10.1038 / \mathrm{nrg} 1348$.

Freckleton RP, Harvey PH, Pagel M. 2002. Phylogenetic analysis and comparative data: a test and review of evidence. American Naturalist 160:712-726 DOI 10.1086/343873.

Hancock JM. 1996. Simple sequences and the expanding genome. BioEssays 18:421-425 DOI 10.1002/bies.950180512.

Harr B, Schlotterer C. 2000. Long microsatellite alleles in Drosophila melanogaster have a downward mutation bias and short persistence times, which cause their genome-wide underrepresentation. Genetics 155: 1213-1220.

Jarvis ED, Mirarab S, Aberer AJ, Li B, Houde P, Li C, Ho SYW, Faircloth BC, Nabholz B, Howard JT, Suh A, Weber CC, da Fonseca RR, Li JW, Zhang F, Li H, Zhou L, Narula N, Liu L, Ganapathy G, Boussau B, Bayzid MS, Zavidovych V, Subramanian S, Gabaldon T, Capella-Gutierrez S, Huerta-Cepas J, Rekepalli B, Munch K, Schierup M, Lindow B, Warren WC, Ray D, Green RE, Bruford MW, Zhan XJ, Dixon A, Li SB, Li N, Huang YH, Derryberry EP, Bertelsen MF, Sheldon FH, Brumfield RT, Mello CV, Lovell PV, Wirthlin M, Schneider MPC, Prosdocimi F, Samaniego JA, Velazquez AMV, AlfaroNunez A, Campos PF, Petersen B, Sicheritz-Ponten T, Pas A, Bailey T, Scofield P, Bunce M, Lambert DM, Zhou Q, Perelman P, Driskell AC, Shapiro B, Xiong ZJ, Zeng YL, Liu SP, Li ZY, Liu BH, Wu K, Xiao J, Yinqi X, Zheng QM, Zhang Y, Yang HM, Wang J, Smeds L, Rheindt FE, Braun M, Fjeldsa J, Orlando L, Barker FK, Jonsson KA, Johnson W, Koepfli KP, O'Brien S, Haussler D, Ryder OA, Rahbek C, Willerslev E, Graves GR, Glenn TC, McCormack J, Burt D, Ellegren H, Alstrom P, Edwards SV, Stamatakis A, Mindell DP, Cracraft J, Braun EL, Warnow T, Jun W, Gilbert MTP, Zhang GJ. 2014. Whole-genome analyses resolve early branches in the tree of life of modern birds. Science 346: 1320-1331 DOI 10.1126/science.1253451. 
352 Jetz W, Thomas GH, Joy JB, Hartmann K, Mooers AO. 2012. The global diversity of birds

353

354

355

356

357

358

359

360

361

362

363

364

365

366

367

368

369

370

371

372

373

374

375

376

377

378

379

380

381 in space and time. Nature 491:444-448. DOI 10.1038/nature11631.

Kofler R, Schlötterer C, Lelley T. 2007. SciRoKo: a new tool for whole genome microsatellite search and investigation. Bioinformatics 23:1683-1685 DOI 10.1093/bioinformatics/btm157.

Kornberg A, Bertsch LL, Jackson JF, Khorana HG. 1964. Enzymatic synthesis of deoxyribonucleic acid: XVI. Oligonucleotides as templates and the mechanisms of their replication. Proceedings of the National Academy of Sciences of the United States of America 51:315-323 DOI 10.1073/pnas.51.2.315.

Kruglyak S., Durrett R., Schug M. D., Aquadro C. F. 2000. Distribution and abundance of microsatellites in the yeast genome can be explained by a balance between slippage events and point mutations. Molecular Biology and Evolution 17:1210-1219 DOI org/10.1093/oxfordjournals.molbev.a026404.

Li WH, Ellsworth DL, Krushkal J, Chang BH, Hewett-Emmett D. 1996. Rates of nucleotide substitution in primates and rodents and the generation-time effect hypothesis. Molecular Phylogenetics and Evolution 5:182-187 DOI 10.1006/mpev.1996.0012.

Masters BS, Johnson LS, Johnson BGP, Brubaker JL, Sakaluk SK, Thompson CF. 2011. Evidence for heterozygote instability in microsatellite loci in house wrens. Biology Letters 7:127-130 DOI 10.1098/rsbl.2010.0643.

Mindell DP, Knight A, Baer C, Huddleston CJ. 1996. Slow rates of molecular evolution in birds and the metabolic rate and body temperature hypotheses. Molecular Biology and Evolution 13:422-426 DOI 10.1093/oxfordjournals.molbev.a025601.

Nabholz B, Glémin S, Galtier N. 2008. Strong variations of mitochondrial mutation rate across mammals - the longevity hypothesis. Molecular Biology and Evolution 25:120-130 DOI $10.1093 / \mathrm{molbev} / \mathrm{msm} 248$.

Ohta T, Kimura M. 1973. A model of mutation appropriate to estimate the number of electrophoretically detectable alleles in a finite population. Genetics Research 22: 201-204 DOI 10.1017/S0016672308009531.

Paradis E, Claude J, Strimmer K. 2004. APE: analysis of phylogenetics and evolution in R language. Bioinformatics 20: 289-290 DOI 10.1093/bioinformatics/btg412. 
382

383

384

385

386

387

388

389

390

391

392

393

394

395

396

397

398

399

400

401

402

403

Qi WH, Jiang XM, Du LM, Xiao GS, Hu TZ, Yue BS, Quan QM. 2015. Genome-wide survey and analysis of microsatellite sequences in bovid species. PLOS ONE 10: e0133667 DOI 10.1371/journal.pone.0133667.

Sturzeneker R, Haddad LA, Bevilacqua RAU, Simpson AJG, Pena SDJ. 1998. Polarity of mutation in tumor-associated microsatellite instability. Human Genetics 102:231-235 DOI $10.1007 / \mathrm{s} 004390050684$.

Taylor JS, Durkin JMH, Breden F. 1999. The death of a microsatellite: a phylogenetic perspective on microsatellite interruptions. Molecular Biology and Evolution 16:567-572 DOI 10.1093/oxfordjournals.molbev.a026138.

Tóth G, Gáspári Z, Jurka J. 2000. Microsatellites in different eukaryotic genomes: survey and analysis. Genome Research 10:967-981 DOI 10.1101/gr.10.7.967.

Vowles EJ, Amos W. 2006. Quantifying ascertainment bias and species-specific length differences in human and chimpanzee microsatellites using genome sequences. Molecular Biology and Evolution 23: 598-607 DOI 10.1093/molbev/msj065.

Wang JF, Qi, HG, Li L, Zhang GF. 2014. Genome-wide survey and analysis of microsatellites in the Pacific oyster genome: abundance, distribution, and potential for marker development. Chinese Journal of Oceanology and Limnology 32:8-21 DOI 10.1007/s00343-014-3064-z.

Yamada NA, Smith GA, Castro A, Roques CN, Boyer JC, Farber RA. 2002. Relative rates of insertion and deletion mutations in dinucleotide repeats of various lengths in mismatch repair proficient mouse and mismatch repair deficient human cells. Mutation ResearchFundamental and Molecular Mechanisms of Mutagenesis 499: 213-225 DOI 10.1016/S0027-5107(01)00282-2. 
404 Figure and Table legends

405

406 Table 1: Result for the relationship between average mismatches and body mass fitted in 407 PGLS analyses.

408

409

Figure 1: Regression scatterplot of the inverse of the average mismatches of imperfect SSRs on the log of body mass in whole genome scale.

411

412 Figure 2: Regression scatterplot of the inverse of the average mismatches of imperfect 413 SSRs in five classes of repeat type on the log of body mass.

Figure S1: The script for performing the PGLS analyses in R.

416

Figure S2: Avian phylogeny used in this study. The phylogeny is presented in Nexus format and can be drawn using any standard tree-drawing package such as TreeView.

419

Figure S3: SSR characteristics for motif sizes 1-6 with minimum 3 repeats in 65 birds.

421

422

Figure S4: Imperfect microsatellites as the percentage of genome size of 65 birds. The

abbreviated bird names are shown in the $\mathrm{x}$-axis and the percentages are shown in the $\mathrm{y}$-axis.

424

425

Table S1: A list of the 65 avian species and average adult body mass, GC content, average mismatches of imperfect microsatellites on the whole genome. Species names are abbreviated with four letters; first letter represents the genus name and last three letters represent the species name. For Pelecanus crispus and Podiceps cristatus, we use Pecri and Pocri separately. Mono-, di-, tri-, tetra-, penta- and hexa- are microsatellite types.

430 
431 Table S2: Microsatellite abundance in 65 birds.

432

433 Table S3: Number of imperfect microsatellites in different avian genomes and the 434 percentage of imperfect microsatellites in the corresponding genome.

435

436 Table S4: Motif length and percentage of imperfection of microsatellites in different species.

437 The paired sample $t$-test results are shown below the data tables.

438

439

Table S5: Average length of imperfect microsatellites compared to perfect microsatellites. 440

Table S6: Genomic abundance of microsatellites having a length of 20 bp that either lack 443 mismatch (perfect motifs) or have exactly one mismatch in each locus across species.

Table S7: Number of microsatellites where motif mismatches are either higher or lower than the expected values of mismatches in different bird genomes.

Table S8: Genomic abundance of imperfect microsatellite loci based on length and number 448 of mismatches.

Table S9: Result for the relationship between average mismatches and body mass fitted in 451 PGLS analyses when different parameters were used to search microsatellites in the genomes (minimum score $=15$ and mismatch penalty $=3$; minimum score $=10$ and mismatch penalty $=5$ ). The average mismatches of imperfect microsatellites for the 65 birds are given on the next page below the result table. 


\section{Table $\mathbf{1}$ (on next page)}

Result for the relationship between average mismatches and body mass fitted in PGLS analyses. 
1 Table 1

2

\begin{tabular}{llccccccc}
\hline & & & \multicolumn{7}{c}{ Body mass } & \multicolumn{3}{c}{ GC content } & \\
\cline { 4 - 9 } Type & Model & $\mathbf{R}^{2}$ & $\boldsymbol{\beta} \pm$ SE & $\boldsymbol{t}$ & $\mathbf{P}$ & $\boldsymbol{\beta} \pm \mathbf{S E}$ & $\boldsymbol{t}$ & $\mathbf{P}$ \\
\hline All & $\mathrm{BM}$ & 0.282 & $0.015 \pm 0.003$ & 4.974 & $<0.001$ & & & \\
& $\mathrm{BM}+\mathrm{GC}$ & 0.340 & $0.015 \pm 0.003$ & 5.102 & $<0.001$ & $-2.533 \pm 1.090$ & 2.324 & 0.023 \\
Di & $\mathrm{BM}$ & 0.279 & $0.022 \pm 0.005$ & 4.932 & $<0.001$ & & & \\
& $\mathrm{BM}+\mathrm{GC}$ & 0.332 & $0.022 \pm 0.004$ & 5.033 & $<0.001$ & $-2.940 \pm 1.255$ & 2.342 & 0.022 \\
Tri & $\mathrm{BM}$ & 0.257 & $0.015 \pm 0.003$ & 4.664 & $<0.001$ & & & \\
& $\mathrm{BM}+\mathrm{GC}$ & 0.293 & $0.015 \pm 0.003$ & 4.711 & $<0.001$ & $-2.044 \pm 1.149$ & 1.778 & 0.080 \\
Tetra & $\mathrm{BM}$ & 0.290 & $0.019 \pm 0.004$ & 5.072 & $<0.001$ & & & \\
& $\mathrm{BM}+\mathrm{GC}$ & 0.393 & $0.019 \pm 0.003$ & 5.382 & $<0.001$ & $-4.074 \pm 1.257$ & 3.241 & 0.002 \\
Penta & $\mathrm{BM}$ & 0.123 & $0.011 \pm 0.004$ & 2.972 & 0.004 & & & \\
& $\mathrm{BM}+\mathrm{GC}$ & 0.205 & $0.011 \pm 0.004$ & 3.050 & 0.003 & $-3.272 \pm 1.296$ & 2.525 & 0.014 \\
Hexa & $\mathrm{BM}$ & 0.254 & $0.013 \pm 0.003$ & 4.634 & $<0.001$ & & & \\
& $\mathrm{BM}+\mathrm{GC}$ & 0.268 & $0.013 \pm 0.003$ & 4.620 & $<0.001$ & $-1.129 \pm 1.049$ & 1.076 & 0.286 \\
\hline
\end{tabular}

3

4 Key to symbols: All, all imperfect microsatellites; Di, Tri, Tetra, Penta, Hexa means imperfect 5 microsatellites with different repeat type; BM, Body mass; GC, GC content.

6

7 


\section{Figure 1 (on next page)}

Regression scatterplot of the inverse of the average mismatches of imperfect SSRs on the log of body mass in whole genome scale. 


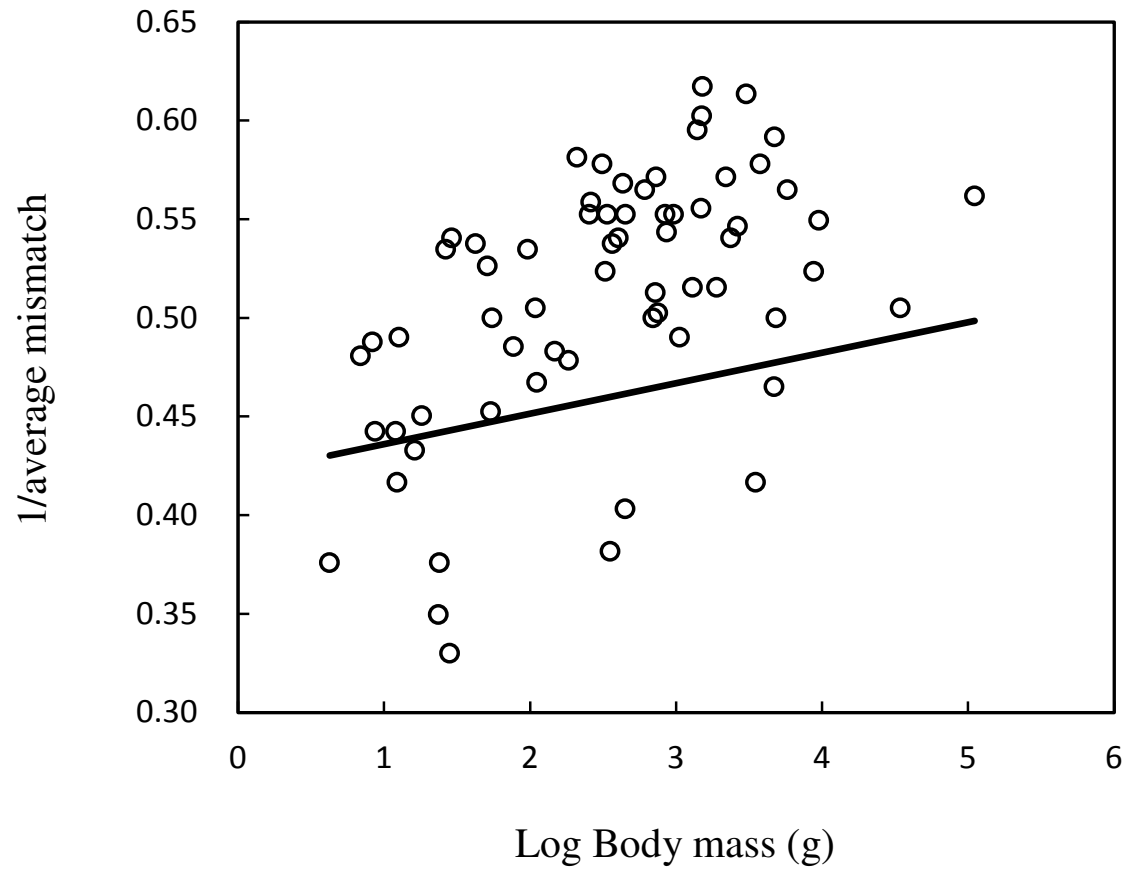


Figure 2 (on next page)

Regression scatterplot of the inverse of the average mismatches of imperfect SSRs in five classes of repeat type on the log of body mass. 


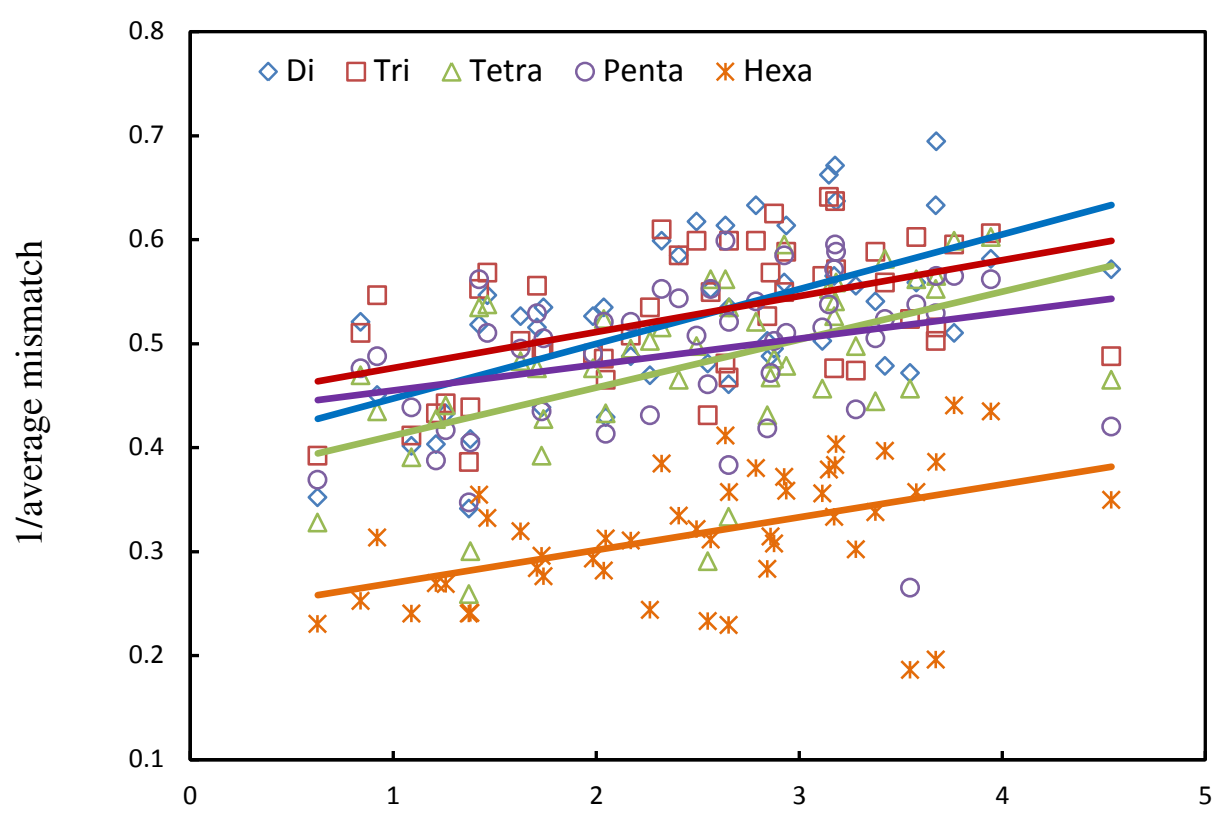

Log Body mass (g) 